\title{
Pisa Syndrome in Parkinson's Disease: A Novel Rehabilitation Approach in a Microgravitary Environment
}

\author{
Raoul Saggini ${ }^{1}$, Teresa Paolucci ${ }^{1 *}$, Claudia Barbato ${ }^{2}$ and Rosa Grazia Bellomo ${ }^{2}$ \\ ${ }^{1}$ Department of Medical and Oral Sciences and Biotechnologies, Unit of Physical Medicine and Rehabilitation, University G. d' Annunzio Chieti, Chieti-Pescara, \\ Italy \\ ${ }^{2}$ Department of Biomolecular Sciences, University of Study of Urbino Carlo Bo, Urbino, Italy
}

*Corresponding author: Teresa Paolucci, University G. d' Annunzio Chieti, Department of Medical and Oral Sciences and Biotechnologies, Viale Abruzzo 322, 66100 CH, Chieti-Pescara, Italy.
Received Date: May 12, 2020

Published Date: June 22, 2020

\section{Abstract}

Background: Pisa Syndrome (PS) is clinically defined as the sustained lateral bending of the trunk and worsened by prolonged sitting or walking.

Aim of the study: To measure the effect of postural exercises under microgravity conditions using the Dynamic-Antigravity-Postural-System (SPAD) and focal mechanical vibration (ViSS) in PS patients.

Materials and methods: A proof concept study was performed. Twenty ( $N=20)$ (mean age $68 \pm 8,4$ years) consecutive patients affected by Pisa Syndrome in Parkinson's Disease with trunk lateral flexion were included. Patients were divided into two groups of 10 patients according to electromyography characteristics: neurogenic group (hyperactivity of lumbar paraspinals ipsilateral to the trunk leaning side) and myogenic group (hyperactivity of paraspinals contralateral to the trunk leaning side).

Results: We found improvements especially in myogenic group, in terms of decrease of lateral trunk flexion $(61 \%$, compared to $35 \%$ of the neurogenic group) and in terms of improvement in paraspinal EMG measurement (43\% compared to $14 \%$ of the neurogenic group) after rehabilitation treatment (T1) respect baseline (T0).

Conclusion: This study suggested the efficacy of specific postural training in a microgravitary environment with SPAD system in combination with focal mechanical vibration as an integrative rehabilitation program in Pisa Syndrome patients.

Keywords: Exercise, Rehabilitation, Focal mechanical vibration, Dynamic-Antigravity-Postural-System (SPAD), Electromyography

\section{Introduction}

The sustained lateral bending of the trunk, associated or not with rotation of the spine along the sagittal plane, is often referred to as Pisa syndrome (PS) [1]. Pisa syndrome is defined as $>10^{\circ}$ lateral flexion in the standing position [2], and it has been suggested that in the context of Parkinson's disease (PD) it is an axial dystonia analogous to that seen as a rare consequence of neuroleptic therapy [3].
PS was originally described by Ekbom (1972) in psychiatric patients: the term was subsequently applied to patients with Alzheimer's disease with and without neuroleptic exposure, in patients with Lewy body dementia [4,5] and in patients on antiemetics and cholinesterase inhibitors [6].

Lateral trunk flexion has also been reported in idiopathic PD patients [7-9] in the absence of treatment with antipsychotics, 
antiemetics, or cholinesterase inhibitors. In 2008 Benatru et al. [10] analyzed the different postural abnormalities in PD [11,12] and concluding that there are several factors that can contribute to their initiation, including disorders of the reflections postural, sensory deficits, lack of correct posture, coordination difficulties or postural balance. However, doubtful correlation remaines between the loss of orientation in postural control and the control of postural stabilization [13-18].

Abnormal postures with lateral flexion in PD are classified into two types on the basis of the pattern of appearance: chronic and sub-chronic. The chronic type of lateral flexion in PD appears subclinically and worsens gradually; such worsening is related to the progression of the disease. The sub-chronic type of lateral flexion in PD is observed in cases reported by Cannas et al. and Gambarin et al. and seems to show similar symptoms with those of Pisa syndrome. The severity of this lateral flexion progresses in a few months and this ipsilateral deviation and a scoliosis-like skeletal deformity were observed, and the severity of this scoliosis was closely related to a decrease in extracellular striatal dopamine level [19-21].

The term Pisa syndrome should not be indiscriminately used for all cases of lateral flexion in PD, and the type of lateral flexion in PD should be carefully observed.

Although a dopaminergic dysfunction or an imbalance in the cholinergic-dopaminergic central pathway have been implicated as explanations for this syndrome [22], the pathophysiology of PS remaines largely unexplained. Some authors consider lateral trunk flexion in PD and levodopa-responding parkinsonism as a trunk dystonia, although electromyographic (EMG) recordings yielded contradictory results [23-28].

To date, there are no definitive rehabilitation protocols in the PS.

So, considering these premises, the purpose of our study was to evaluate the effect of postural exercises under microgravity conditions using the Dynamic-Antigravity-Postural-System (SPAD) and focal mechanical vibration (ViSS) in PS patients.

\section{Materials and Methods}

A proof concept study was performed. Twenty $(\mathrm{N}=20)$ (mean age $68 \pm 8,4$ years) consecutive patients affected by Pisa Syndrome in Parkinson's Disease with right trunk lateral flexion were enrolled. Patients were community-dwelling people affected by Parkinson's Disease (PD) associated with right lateral trunk flexion (LTF).

Patients were eligible to participate if they were aged $\geq 45$ years of age and $\leq 80$ years of age and they fulfilled United Kingdom Parkinson's Disease Society Brain Bank clinical diagnostic criteria for PD, their Mini-Mental State Examination (MMSE) scores was $\geq$ 24 and Hoehn- Yahr stage $>1$ and $<4$.
Patients were excluded if they had symptoms and compatible signs with atypical parkinsonism; If they intake of central dopamine receptor antagonists during the six months preceding the enrollment (first and second-generation antipsychotics with the exception of clozapine and quetiapine, antiemetics, prokinetics; if they had positive history for major surgeries to the spine (tumors, infections, ankylosing spondylitis, paraneoplastic syndromes) or they suffered of other neurological diseases.

The electromyographic examination (EMG) was performed at the baseline to detect the patterns of activation of the paravertebral muscles of the trunk in a population of patients affected by Parkinson's disease (PD) complicated by Pisa Syndrome (PS), to measure the effect of a complex postural rehabilitation treatment evaluated in terms of reduction of the EMG activation pattern.

Also, PDQ-8 a short form of the PDQ-39, the PDQ-8 has been developed using questions taken from the larger instrument to provide a single index score that is almost identical to those gained from the PDQ-39. The PDQ-8 provides a reliable measure of overall health status and is ideal for studies in which a shorter questionnaire is preferred. Moreover, UPDRS scale was performed: it has four components, largely derived from pre-existing scales that were reviewed and modified by a consortium of movement disorders specialists (Part I, Mentation, Behavior and Mood; Part II, Activities of Daily Living; Part III, Motor; Part IV, Complications). Specific anamnesis for PS (age of onset, side bending, side and type of hyperactivity at EMG tracing were assessed. The presence of back pain and its intensity measured by the Visual Analogue Scale (VAS) was considered. The clinical evaluation also included accurate testing of muscle mass, strength, and range of motion. The clinical evaluation of PS was performed through a wall goniometer, which allowed the measurement of the inclination angle in the frontal plane, and through an electromyograph, which recorded the paraspinal muscles activity. We performed conventional EMG investigation. Electromyography (EMG) [29-31] deals with the study of muscular and motor unit potentials (MUP) recorded with a needle electrode inserted into the muscle. It uses disposable needle electrodes which record the activity of muscle fibers in different positions - at rest, during a voluntary and progressive contraction until maximum effort and during a sustained contraction. In this case, we inserted the electrodes within the paravertebral muscles at thoracic-lumbar levels T4-T6, T6-T8, T8-T10 and T12-L1, evaluating the following positions with a score ranging from -3 (for "no contraction") to +3 (for "strong activity"): standing, left and right lateral bending, flexion and extension. Likewise, we detected the possible presence of muscular co-contraction. The resulting morphology was stratified into i) neurogenic, ii) myogenic or iii) normal tracing.

The patients were divided in two different groups, according to their EMG tracing (neurogenic/myogenic - see Table 1). 
Table 1: Characteristics of patients.

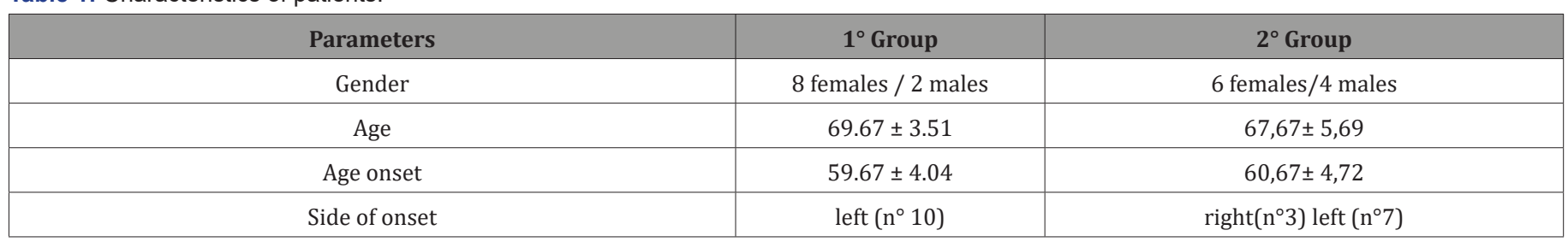

In all cases, PD onset affected the left hemi soma with a tremor phenotype. EMG muscular hyperactivity was contralateral to the bending side, which resulted in a myogenic tracing. All patients underwent a cycle of ten 90-minute sessions of complex postural rehabilitation treatment, three times for week with SPAD system and focal mechanical vibration of trunk muscles by Viss stimulation at $300 \mathrm{~Hz}$ associated to a "trunk-specific rehabilitation program"[32-36] composed of exercises depending on the single patient's motor and functional status:

\section{Stretching exercises}

Exercises to stretch the muscles of the posterior kinematic chain; exercises to stretch the pectoralis muscles; exercises to stretch the hamstring muscles; exercises to stretch lumbar and dorsal muscles; diaphragmatic deconditioning in the supine position.

\section{Relaxation exercises}

Segmental passive mobilization -until maximum joint range of motion is reached-; breathing exercises to promote expansion of the chest). All patients were evaluated before starting the rehabilitative protocol (T0) and immediately after the end of the treatment (T1). the Dynamic Antigravity Postural System (SPAD): the SPAD is a device for bodyweight relief, consisting of a machine that is designed to reduce, modify, and condition the force of gravity that acts on the body structures of movement during rectilinear motion. This system is based on the rationale that gait training can combine a motor task with sensory feedback, consistent with the multisensory approach to postural control in body's segment aligment. The SPAD system is composed of a treadmill on which the patient trains, supporting his body weight, and a structure to which he is harnessed with a pneumatic belt that us placed between the iliac crests and costal arches, connected to a lifting system with 4 tie-rods that attached to the body and pelvic girdle this equipment is completed by 4 front pads ( 2 on the humeral heads for the shoulder girdle and 2 on the anterior superior iliac spine for the pelvis) that act as stabilizers and proprioceptive stimulus, preventing twisting of the pelvis or shoulder during movement on the treadmill, and as proprioceptive informants and 2 rear pads, one placed on between scapulae region and one on the sacral apex.

\section{Statistical analysis}

Statistical analysis was conducted with the SPSS software package for Windows, version 19.0. The statistical analysis of the continuous variables was conducted calculating median and range (min-max), because these variables were not normally distributed. To evaluate the functional scales adopted we used the Friedman test for paired samples. The significance level was set at $<0.05$.

\section{Results}

An improvement especially in myogenic group, in terms of decrease of lateral trunk flexion (61\%, compared to $35 \%$ of the neurogenic group) and in terms of improvement in paraspinal EMG measurement ( $43 \%$ compared to $14 \%$ of the neurogenic group) after rehabilitation treatment (T1) respect baseline (T0), was observed respect to the evaluation scales. EMG muscular hyperactivity was contralateral to the bending side, which resulted in a myogenic tracing, instead EMG muscular hyperactivity was ipsilateral to the bending side, which resulted in a neurogenic tracing. EMG on the cervical, thoracic and lumbar paraspinal muscles (see Figure 1-6).

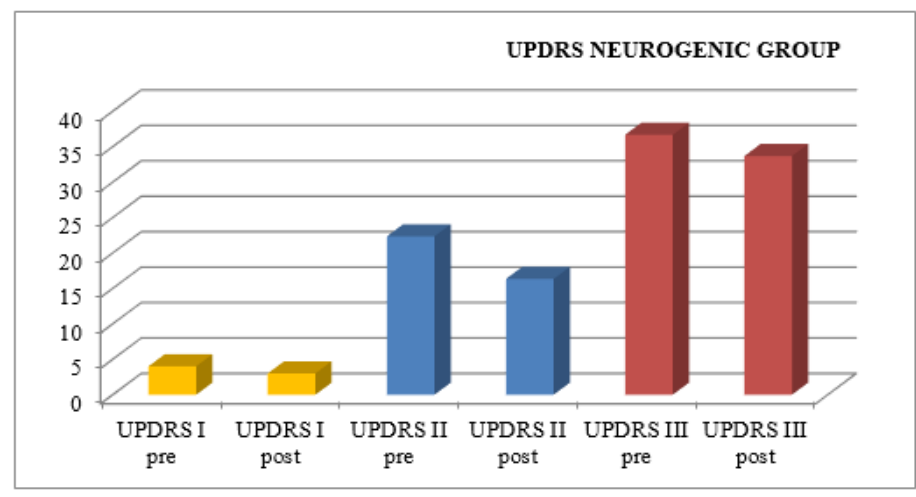

Figure 1: UPDRS results in NEUROGENIC GROUP. 


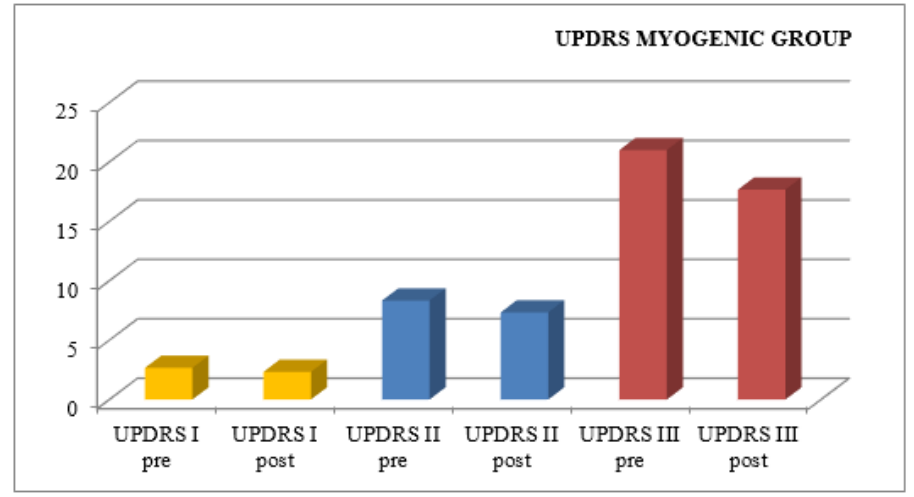

Figure 2: UPDRS results in MYOGENIC GROUP (Pre= T0; Post= T1).

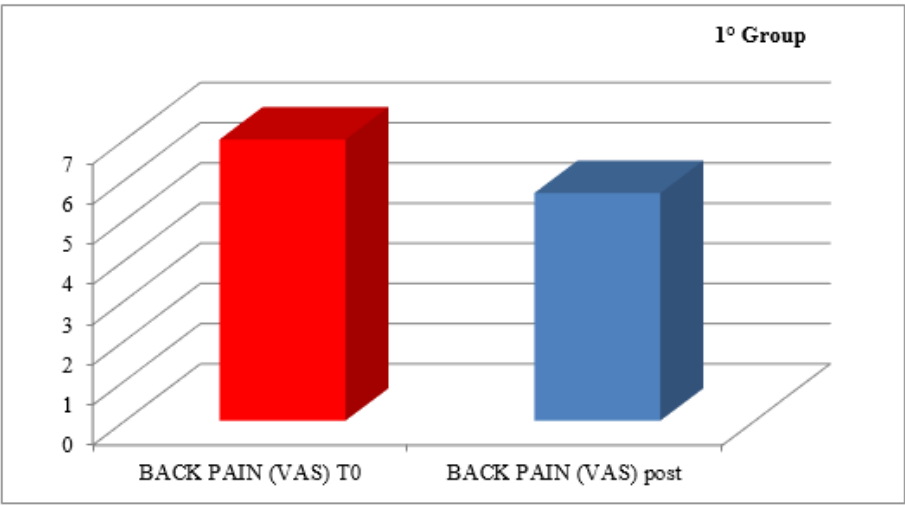

Figure 3: VAS results in $1^{\circ}$ GROUP (T0 vs post=T1).

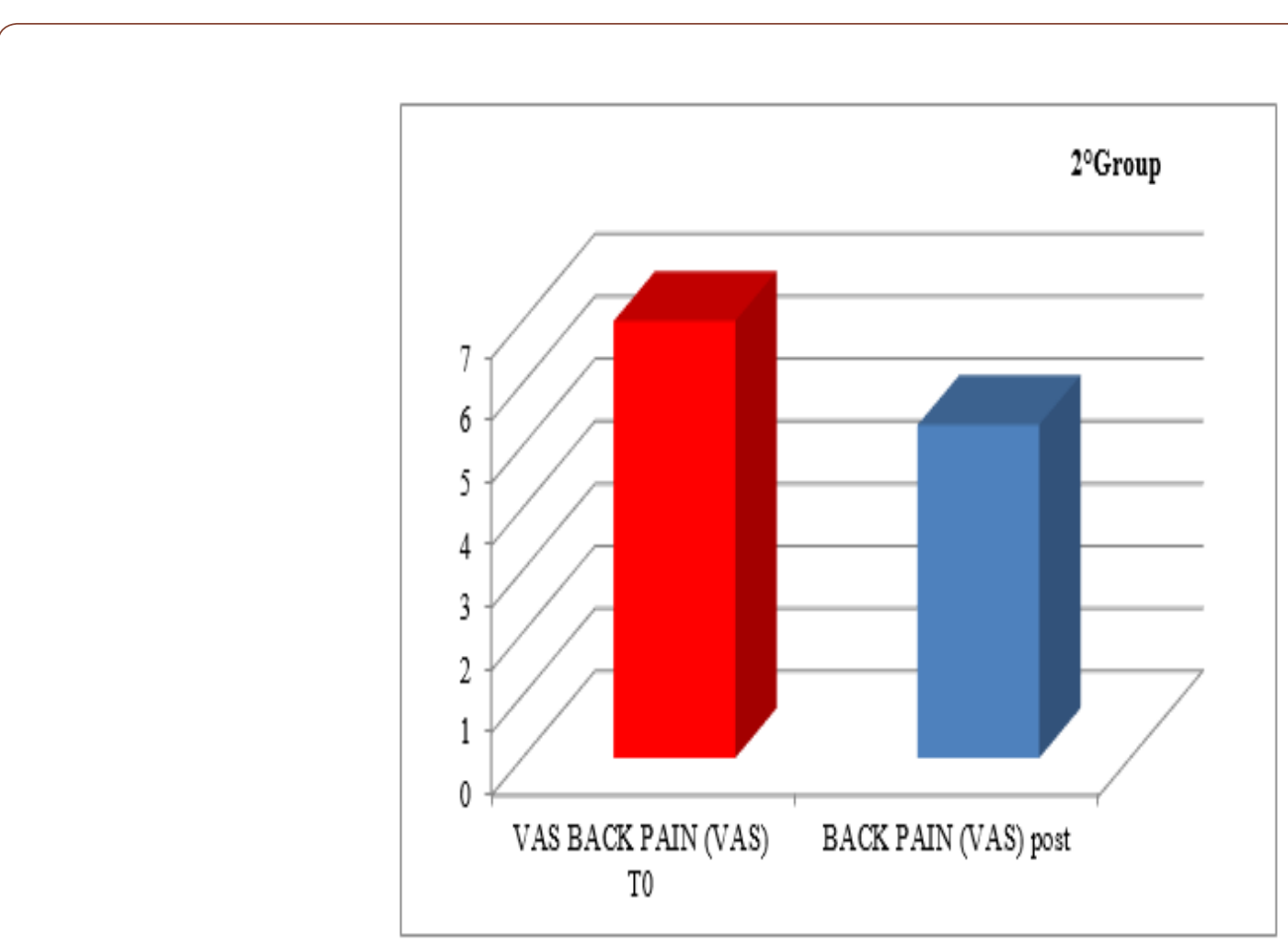

Figure 4: VAS results $2^{\circ}$ GROUP (T0 vs post=T1). 


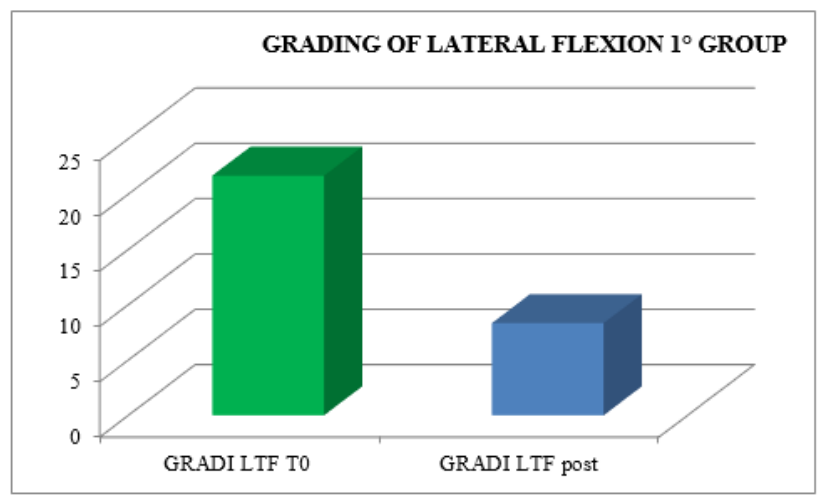

Figure 5: Grading of lateral flexion for $1^{\circ}$ GROUP (T0 vs post=T1).

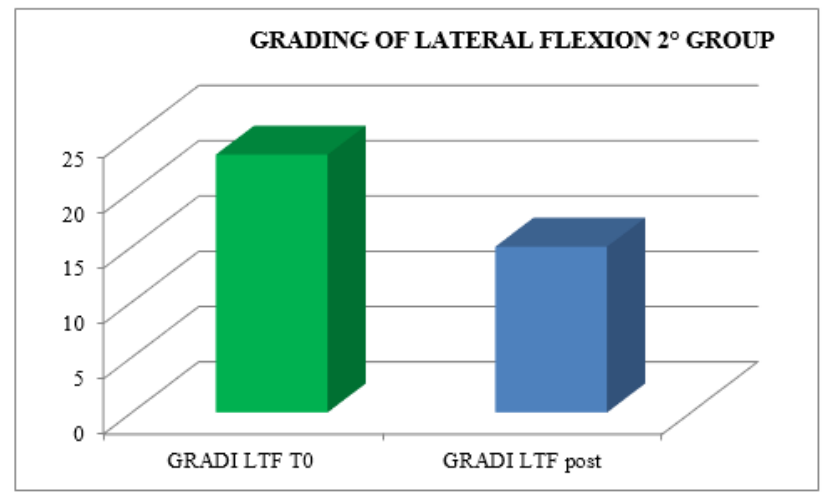

Figure 6: Grading of lateral flexion for $2^{\circ}$ GROUP (T0 vs post=T1).

\section{Discussion}

The postural complex rehabilitation treatment proposed to the PS patients in this study showed a good result respect to the gradual improvement of the trunk flexibility and the clinical status of PD patients with Pisa Syndrome, acknowledging the precocity of the identification of a trunk lateral deviation as a crucial prognostic and therapeutic stage. All patients reported a significant clinical improvement. In particular, patients with a myogenic tracing reported an important improvement in the EMG measurement carried out in standing position and at rest, as well as in the intensity of contraction during the right lateral flexion, which led to a recruitment of the paravertebral muscles previously traced as myogenic. Likewise, the clinical improvement, in terms of reduction latero-flexion degrees, was favorable for patients with a myogenic tracing. Unlikely, patients with neurogenic tracing did not report statistically relevant improvements from the EMG point of view. A considerable reduction of back pain that indirectly eases the performance of daily life activities was observed in the two groups and, to a lesser extent, patients with myogenic tracing also recorded an improvement of back pain reduction. We can hypothesize that the marked improvement of some variables in the group of patients with myogenic tracing is attributable to a modulation of rheological characteristics of muscle related to Viss treatment with the increased responsiveness of the muscles during its elongation; instead the smaller variables in the group of patients with neurogenic tracing is attributable to a modulation of rheological characteristics of muscles related to Viss treatment but in a context of the fibrotic and less elastic consistency of a neurogenic muscle. A further hypothesis lies in the concept that patients with neurogenic tracing may have a right lateral trunk flexion structured in a dismorphism, whereas patients with myopathic tracing have a lateral trunk flexion structured in a paramorfism, which therefore can be modified with mechano-acoustic vibration and postural exercise with SPAD corrections [37].

Moreover, it is common knowledge that, during the postural treatment, the release and the elongation of the diaphragm and the psoas muscle determine an improvement of the spine range of motion. The lengthening of the posterior kinetic chain allows, in any case, the increase of blood flow with subsequent changes of tissue oxygenation and the release of endorphins, which are all factors that positively affect the pain.

In accordance with the 2010 study conducted by Di Matteo and Fasano38, the work confirms that PS is not exclusively a form of dystonia, but it can result in two different pathophysiological 
mechanisms that we defined as myogenic and neurogenic. They found paraspinal muscle hyperactivity more frequently contralateral to the leaning side and, in only a minority of patients, with a typical dystonic pattern. In our paradigm, EMG investigation of paraspinal muscles detected hyperactivity consistently on the side of bending at the thoracic level. At the lumbar level, we obtained contradictory results, which may be explained by the muscle misuse caused by the chronic forced fixed posture of the trunk. This hypothesis was also suggested by the atrophic pattern of muscles at the lumbar level, more marked on the leaning side.

Although only slightly significant, the correlation that emerged between lateral trunk flexion and the UPDRS-III score suggests that axial deviation may contribute to determining the extent of the motor impairment in PD patients. This latter observation is further supported by the finding of strong correlations between postural (lateral trunk flexion) and motor (limited range of trunk flexion and inclination) abnormalities of the trunk. Taken together, these results suggest that PD patients with lateral trunk flexion can be considered a functional subgroup requiring specific rehabilitation management. This view is supported by the findings of other researchers who suggested that patients with trunk abnormalities showing axial rigidity, gait disorder, postural instability, and poor L-dopa responsiveness are probably a group affected by a selective form of PD who would benefit greatly from an additional, nonpharmacological treatment strategy [38].

\section{Conclusion}

Rehabilitation interventions represent an alternative strategy to help pharmacological treatment in order to slow or reverse some functional aspects of disability in Parkinson's disease and we posit that our sensorimotor rehabilitation protocol could represent a valid effective rehabilitation path in patients with Pisa PD syndrome.

Despite the benefits produced by the complex rehabilitation treatment, these preliminary data represent only a trend, and therefore, they require an in depth examination of a wider number of patients and a more extended follow-up period, in order to confirm the results in the medium and long term.

\section{Acknowledgement}

None.

\section{Conflict of Interest}

All authors declare no potential conflict of interest and this research was not supported by any funding sources

\section{References}

1. Tassorelli C, Furnari A, Buscone S, Alfonsi E, Pacchetti C, et al. (2012) Pisa Syndrome in Parkinson's Disease: Clinical, Electromyographic,and Radiological Characterization. Mov Disord 27(2): 227-235.

2. Doherty KM, van de Warrenburg BP, Peralta MC, Moriyama LS, Azulay JP, et al. (2011) Postural deformities in Parkinson's disease. Lancet Neurol $10(6): 538-549$

3. Ekbom K, Lindholm H, Ljungberg L (1972) New dystonic syndrome associated with butyrophenone therapy. Z Neurol 202(2): 94-103.

4. Davidson M, Powchilk P, Davis KL (1988) Pisa syndrome in Alzheimer's disease. Biol Psychiatry 23(2): 213.

5. Gibb WR, Lees AJ (1988) The relevance of the Lewy body to the pathogenesis of idiopathic Parkinson's disease. J Neurol Neurosurg Psychiatry 51(6): 745-752.

6. Suzuki T, Hori T, Baba A, Abe S, Shiraishi H, et al. (1999) Effectiveness of anticholinergics and neuroleptic dose reduction on neuroleptic-induced pleurothotonus (the Pisa syndrome). J Clin Psychopharmacol 19(3): 277-280.

7. Cannas A, Solla P, Floris G, Borghero G, Tacconi P, et al. (2005) Reversible Pisa syndrome in Parkinson's disease during treatment with Pergolide: a case report. Clin Neuropharm 28(5):252-253.

8. Di Matteo A, Fasano A, Squintani G, Ricciardi L, Bovi T, et al. (2011) Lateral trunk flexion in Parkinson's disease: EMG features disclose two different underlying pathophysiological mechanism. J Neurol 258(8): $740-745$.

9. Gambarin M, Antonini A, Moretto G, Bovi P, Romito S, et al. (2006) Pisa syndrome without neuroleptic exposure in a patient with Parkinson's disease: case report. Mov Disord 21(2): 270-273.

10. I Benatru, M Vaugoyeau, JP Azulay (2008) Postural disorders in Parkinson's disease. Neurophysiol Clin 38(6): 459-465.

11. Gambarin M, Antonini A, Moretto G, Bovi P, Romito S, et al. (2006) Pisa syndrome without neuroleptic exposure in a patient with Parkinson's disease: case report. Mov Disord 21(2): 270-273.

12. Kashihara K, Ohno M, Tomita S (2006) Dropped head syndrome in Parkinson's disease. Mov Disord 21(8): 1213-1216.

13. Kitamura J, Nakagawa H, Linuma K, Kobayashi M, Okauchi A, et al. (1993) Visual influence on center of contact pressure in advanced Parkinson's disease. Arch Phys Med Rehabil 74(10): 1107-1112.

14. J Stozek, M Rudzinska, K Longawa, A Szczudlik (2003) The effect of the complex rehabilitation on posture and gait in Parkinson Disease. Neurol Neurochir Pol 7(Suppl 5): 67-81.

15. A Cannas, P Solla, G Floris, P Tacconi, A Serra et al. (2009) Reversible Pisa syndrome in patients with Parkinson's disease on dopaminergic therapy. J Neurol 256(3): 390-395.

16. A Villarejo, A Camacho, R Garcia-Ramos, $\mathrm{T}$ Moreno, M Penas, et al. (2003) Cholinergic-dopaminergic imbalance in Pisa syndrome. Clin Neuropharmacol 26(3): 119-121.

17. Cannas A, Solla P, Floris G, Borghero G, Tacconi P, et al. (2005) Reversible Pisa syndrome in Parkinson's disease during treatment with Pergolide: a case report. Clin Neuropharm 28(5):252-253.

18. F Yokochi (2006) Lateral flexion in Parkinson's disease and Pisa syndrome. J Neurol 253(Suppl 7): VII 17-VII 20.

19. Slawek J, Derejko M, Lass P, Dubaniewicz M (2006) Camptocormia or Pisa syndrome in multiple system atrophy. Clin Neurol Neurosurg 108(7): 699-704.

20. Ekbom K, Lindholm H, Ljungberg L (1972) New dystonic syndrome associated with butyrophenone therapy. Z Neurol 202(2): 94-103.

21. Davidson M, Powchilk P, Davis KL (1988) Pisa syndrome in Alzheimer's disease. Biol Psychiatry 23(2): 213.

22. A Villarejo, A Camacho, R Garcia-Ramos, T Moreno, M Penas, et al. (2003) Cholinergic-dopaminergic imbalance in Pisa syndrome. Clin Neuropharmacol 26(3): 119-121.

23. Di Matteo A, Fasano A, Squintani G, Ricciardi L, Bovi T, et al. (2011) Lateral trunk flexion in Parkinson's disease: EMG features disclose two different underlying pathophysiological mechanism. J Neurol 258(8): 740-745. 
24. Delli Pizzi S, Bellomo RG, Carmignano SM, Ancona E, Franciotti R, et al. (2017) Rehabilitation program based on sensorimotor recovery improves the static and dynamic balance and modifies the basal ganglia neurochemistry: A pilot 1H-MRS study on Parkinson's disease patients. Medicine (Baltimore) 96(50): e8732.

25. Bartolo M, Serraro M, Tassorelli C, Don R, Ranavolo A, et al. (2010) Fourweek trunk specific rehabilitation treatment improves Lateral Trunk Flexion in Parkinson's disease. Mov Disord 25(3): 325-331.

26. SS Palmer, JA Mortimer, DD Webster, R Bistevins, GK Dickson (1986) Exercise therapy for Parkinson's disease. Arch Phys Med Rehabil 67(10): 741-745.

27. Paolucci T, Zangrando F, Piccinini G, Deidda L, Basile R, et al. (2017) Impact of Mézières Rehabilitative Method in Patients with Parkinson's Disease: A Randomized Controlled Trial. Parkinsons Dis 2017: 2762987.

28. C Vitale, V Marcelli, T Furia, Santangelo G, Cozzolino A, et al. (2011) Vestibular impairment and adaptive postural imbalance in Parkinsonian patients with Lateral Trunk Flexion. Mov Disord 26(8): 1458-1463.

29. Frazzitta G, Balbi P, Gotti F, Maestri R, Sabetta A, et al. (2015) Pisa Syndrome in Parkinson's Disease: Electromyographic Aspects and Implications for Rehabilitation. Parkinsons Dis 2015: 437190.

30. Di Matteo A, Fasano A, Squintani G, Ricciardi L, Bovi T, et al. (2011) Lateral trunk flexion in Parkinson's disease: EMG features disclose two different underlying pathophysiological mechanism. J Neurol 258(8): $740-745$.
31. F Yokochi (2006) Lateral flexion in Parkinson's disease and Pisa syndrome. J Neurol 253(Suppl 7): VII 17-VII 20.

32. Zangrando F, Piccinini G, Pelliccioni A, Saraceni VM, Paolucci T (2015) Neurocognitive rehabilitation in Parkinson's disease with motor imagery: a rehabilitative experience in a case report. Case Rep Med 2015: 670385 .

33. Schenkman M, Cutson TM, Kuchibhatla M, J Chandler, CF Pieper, et al. (1998) Exercise to improve spinal flexibility and function for people with Parkinson's disease-a randomized, controlled trial. J Am Geriatr Soc 46(10): 1207-1216.

34. Viliani T, Pasquetti P, Magnolfi S, Lunardelli ML, Giorgi C, et al. (1999) Effects of physical training on straightening up processes in patients with Parkinson's disease. Disabil Rehabil 21(2): 68-73.

35. Oddsson LI (1990) Control of voluntary trunk movements in man. Mechanisms for postural equilibrium during standing. Acta Physiol Scand Suppl 595: 1-60.

36. Bartolo M, Serraro M, Tassorelli C, Don R, Ranavolo A, et al. (2010) Fourweek trunk specific rehabilitation treatment improves Lateral Trunk Flexion in Parkinson's disease. Mov Disord 25(3): 325-331.

37. JS Kim, JW Park, YI Kim, HT Kim, KS Lee (2007) Pisa syndrome as a motor complication of Parkinson's disease. Parkinsonism Relat Disord 13: $126-128$.

38. Lanzetta D, Cattaneo D, Pellegatta D, Cardini R (2004) Trunk control in 